\title{
ULTRASTRUCTURE OF SARS-COV, FIPV, AND MHV REVEALED BY ELECTRON CRYOMICROSCOPY
}

\author{
Benjamin W. Neuman, Brian D. Adair, Craig Yoshioka, Joel D. Quispe, \\ Ronald A. Milligan, Mark Yeager, and Michael J. Buchmeier*
}

\section{INTRODUCTION}

The current understanding of coronavirus ultrastructure relies heavily on transmission electron microscopy of negatively stained images. Such images typically show desiccated specimens and derive contrast from the accumulation of heavy metal negative stains, distorting the sample in the resulting image. Electron cryomicroscopy (cryo-EM) avoids some of the drawbacks of negative staining by imaging frozen specimens preserved in a fully hydrated state in vitreous ice. Cryo-EM images typically derive contrast solely from the density of the imaged sample and the surrounding ice matrix. A limited analysis of porcine transmissible gastroenteritis virus (TGEV) imaged by cryo-EM has been previously reported. ${ }^{1}$ In this report we present a more detailed description of the supramolecular design of three coronaviruses: SARS coronavirus (SARS-CoV), feline infectious peritonitis virus (FIPV), and murine hepatitis virus (MHV).

Coronaviruses are usually classified as non-icosahedral, pleomorphic, enveloped viruses. Cryo-EM has revealed that other pleomorphic viruses have a roughly spherical appearance, studded with projections that correspond to oligomers of the attachment and fusion protein. Examples include influenza virus ${ }^{2-4}$; several retroviruses such as foamy virus, ${ }^{5}$ human immunodeficiency virus, ${ }^{6-10}$ murine leukemia virus, ${ }^{11}$ and Rous sarcoma virus $^{12,13}$; La Crosse virus ${ }^{14,15}$; Sendai virus ${ }^{16}$; and Pichinde, Tacaribe, and lymphocytic choriomeningitis viruses. ${ }^{17}$ Based on single-particle image analysis of arenaviruses imaged by cryo-EM, we have proposed that pleomorphic arenavirus particles are constructed from overlapping paracrystalline lattices of proteins, and that these lattices span the viral membrane. ${ }^{11}$ We hypothesized that coronaviruses may contain a similar supramolecular arrangement of proteins comprising a membrane-proximal scaffold. Here we used cryo-EM to examine the ultrastructure of a selection of coronaviruses representing two of the three proposed phylogenetic groups.

*The Scripps Research Institute, La Jolla, California. 


\section{PARTICLE CHARACTERISTICS}

Particles of SARS-CoV, FIPV, and MHV were prepared from Vero-E6, AK-D, and DBT cells, respectively. MHV and SARS-CoV were also produced in cells cultured with tunicamycin, to form spike-depleted particles with low infectivity. For safety reasons, all particles were fixed with 10\% (for SARS-CoV) or 1\% (for FIPV and MHV) formalin in pH 6.5 HEPES-buffered physiological saline before imaging. All viruses were collected by sucrose gradient ultracentrifugation, and each remained highly infectious until fixed.

Each virus appeared approximately round in cryo-EM images, with a fringe of spikes protruding from the viral membrane and a region of lower density near the virion center (Fig. 1A-B). The average diameter of the membrane-enclosed part of each virus was similar, ranging from $\sim 830 \AA$ for SARS-CoV to $\sim 960 \AA$ for FIPV (Fig. 1C). The diameters of MHV and SARS-CoV virions were distributed more tightly than diameters of FIPV or spike-depleted, tunicamycin-grown MHV. The mean diameters of native and tunicamycin-grown MHV were similar.

Particles of SARS-CoV and MHV produced from tunicamycin-treated cells lacked the characteristic fringe of spikes, but were otherwise indistinguishable from particles grown under standard culture conditions (Fig. 2). Spike-depleted SARS-CoV particles appeared similar to spike-depleted MHV particles in negative stain, but were produced in lower yield, not suitable for effective cryo-EM imaging. Particles were imaged in several degrees of focus in order to emphasize different structural elements. Fine features such as the phospholipids headgroup densities of the viral membrane and individual nucleocapsid protein densities are revealed more clearly in images recorded relatively near to focus (Figs. 1A-B, 2A right). Images recorded farther from focus reveal spikes more clearly at the edge and center of each particle (Fig. 2A, left).

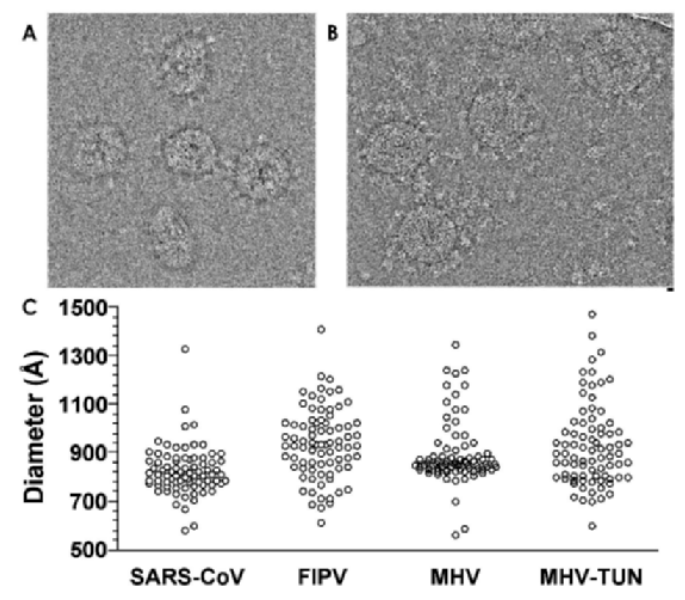

Figure 1. Cryo-EM images of formalin-fixed coronavirus particles in vitreous ice. Images are presented in "reversed contrast" with density depicted in white. Near-to-focus images of fields of SARS-CoV-TOR2 (A) and FIPV-Black (B) virions show pleomorphic enveloped particles with a slight electron-lucent hollow region near the center. Side projections of spikes are visible at the virion edge, and indistinct end-on projections of the spike are located nearer to the virion center. The scale bar below (B) represents $100 \AA$. The membrane-to-membrane diameter of eighty virions was measured from cryo-EM images of SARS-CoV, FIPV, MHV-OBLV60 (MHV), or tunicamycin-grown spike-depleted MHV-OBLV60 (MHV-TUN). 


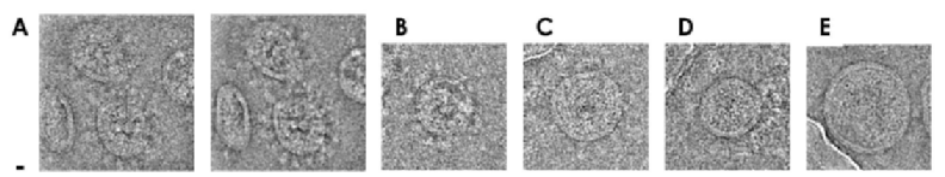

Figure 2. Cryo-EM images of formalin-fixed coronavirus particles in vitreous ice. Close-to-focus images of fields of SARS-CoV-TOR2 (A, $2 \mu \mathrm{m}$ below true focus; B, $4.5 \mu \mathrm{m}$ below focus; C, $2.5 \mu \mathrm{m}$ below focus), FIPVBlack (C, $2.5 \mu \mathrm{m}$ below focus), MHV-OBLV60 (D, $2.5 \mu \mathrm{m}$ below focus), and tunicamycin-grown spikedepleted MHV-OBLV60 (E, $2.5 \mu \mathrm{m}$ below focus).

\section{VIRAL RIBONUCLEOPROTEIN}

Preparations of each virus contained a small amount of material that was consistent with the appearance of coronavirus ribonucleoprotein (RNP). ${ }^{18}$ A particularly interesting image of a SARS-CoV particle trapped in a partially uncoated state at the time of freezing (Fig. 3A-B) shows the spiral RNP partially uncoiled from an approximately round RNP core. The RNP proximal to the extruded membrane segment remains roughly spherical, and appears to be connected to the inner face of the membrane at the ruptured fringe (Fig. 3C).

\section{STRUCTURE OF THE VIRION}

The supramolecular architecture of SARS-CoV, FIPV, and MHV appears quite similar. Each virus is covered with spikes that extend $\sim 200 \AA$ from the peak density of the headgroups in the outer leaflet of the viral membrane. There appears to be a gap between adjacent end-projected spikes near the virion center (Fig. 2A right, for example). The arrangement of spike densities near the center of some particles approximates a rhombus, which would not be inconsistent with a paracrystalline organization of spikes as observed in the virions of pleomorphic arenavirus particles, ${ }^{17}$ or a local hexagonal close-packing of structural proteins as observed in retroviral particles. ${ }^{11}$ Coronavirus particles, as previously reported, appear pleomorphic, and deviate more sharply from a circular profile than other

\section{A}

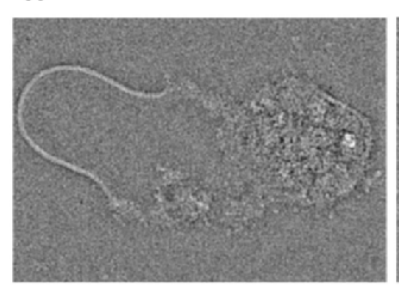

B

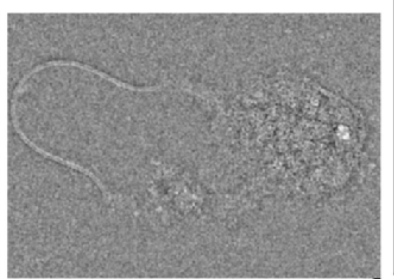

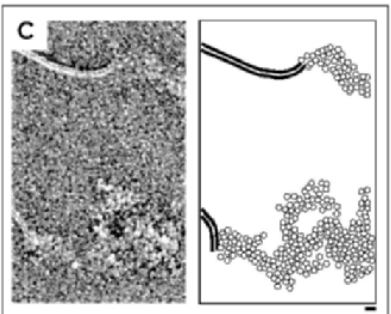

Figure 3. Ribonucleoprotein released from a spontaneously disrupted SARS-CoV particle. The viral ribonucleoprotein is shown at two levels of focus, (A, $4.5 \mu \mathrm{m}$ below true focus; $\mathrm{B}, 2.0 \mu \mathrm{m}$ below true focus). A third image, created by superimposing the low-resolution components of (A) with the high-resolution components of $(\mathrm{B})$ shows the ribonucleoprotein more clearly $(\mathrm{C}$, left). An interpretation of this image $(\mathrm{C}$, right) depicts the viral membrane in bold lines and nucleoprotein molecules as circles. Scale bars denote $100 \AA$. 


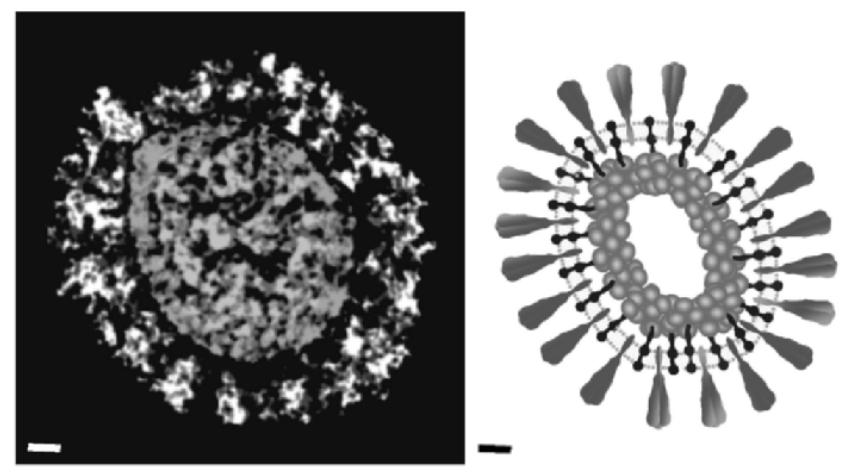

Figure 4. Image of a SARS-CoV particle extracted from the background (left panel) and a schematic crosssectional representation of that part (right panel). Shaded spike proteins are shown at the surface of the virion, with black $\mathrm{M}$ proteins forming a connection to the shaded spiral ribonucleoprotein in the virion core. Scale bars denote $100 \AA$.

pleomorphic virions we have examined by cryo-EM. The observed variability in shape and size of the coronavirus particle would typically be considered inconsistent with icosahedral organization. The observation that the helical RNP is retained in a rough sphere through apparent interaction with proteins resident in the viral membrane is consistent with the spherical arrangement of the viral nucleocapsid proposed for TGEV. ${ }^{19}$ However, further image analysis and biochemical experimentation will be required to determine the supramolecular organization of the virion.

\section{ACKNOWLEDGMENTS}

Some of the work described here was conducted at the National Resource for Automated Molecular Microscopy (NRAMM), which is supported by the National Institutes of Health though the National Center for Research Resources' P41 program (RR17573). This work was supported by NIH grants AI059799, AI025913, and NS41219, and by NIH/NIAID contract HHSN266200400058C.

\section{REFERENCES}

1. C. Risco, I. M. Anton, L. Enjuanes, and J. L. Carrascosa, The transmissible gastroenteritis coronavirus contains a spherical core shell consisting of M and N proteins, J. Virol. 70, 4773-4777 (1996).

2. F. P. Booy, R. W. Ruigrok, and E. F. van Bruggen, Electron microscopy of influenza virus. A comparison of negatively stained and ice-embedded particles, J. Mol. Biol. 184, 667-676 (1985).

3. Y. Fujiyoshi, N. P. Kume, K. Sakata, and S. B. Sato, Fine structure of influenza A virus observed by electron cryo-microscopy, EMBO J. 13, 318-326 (1994).

4. T. Shangguan, D. P. Siegel, J. D. Lear, P. H. Axelsen, D. Alford, and J. Bentz, Morphological changes and fusogenic activity of influenza virus hemagglutinin, Biophys. J. 74, 54-62 (1998).

5. T. Wilk, V. Geiselhart, M. Frech, S. D. Fuller, R. M. Flugel, and M. Lochelt, Specific interaction of a novel foamy virus Env leader protein with the N-terminal Gag domain, J. Virol. 75, 7995-8007 (2001). 
6. J. A. Briggs, T. Wilk, R. Welker, H. G. Krausslich, and S. D. Fuller, Structural organization of authentic, mature HIV-1 virions and cores, EMBO J. 22, 1707-1715 (2003).

7. S. D. Fuller, T. Wilk, B. E. Gowen, H. G. Krausslich, and V. M. Vogt, Cryo-electron microscopy reveals ordered domains in the immature HIV-1 particle, Curr. Biol. 7, 729-738 (1997).

8. T. Goto, T. Ashina, Y. Fujiyoshi, N. Kume, H. Yamagishi, and M. Nakai, Projection structures of human immunodeficiency virus type 1 (HIV-1) observed with high resolution electron cryo-microscopy, J. Electron Microsc. (Tokyo) 43, 16-19 (1994).

9. M. V. Nermut, C. Grief, S. Hashmi, and D. J. Hockley, Further evidence of icosahedral symmetry in human and simian immunodeficiency virus, AIDS Res. Hum. Retroviruses 9, 929-938 (1993).

10. T. Wilk, I. Gross, B. E. Gowen, T. Rutten, F. de Haas, R. Welker, H. G. Krausslich, P. Boulanger, and S. D. Fuller, Organization of immature human immunodeficiency virus type 1, J. Virol. 75, 759-771 (2001).

11. M. Yeager, E. M. Wilson-Kubalek, S. G. Weiner, P. O. Brown, and A. Rein, Supramolecular organization of immature and mature murine leukemia virus revealed by electron cryo-microscopy: implications for retroviral assembly mechanisms, Proc. Natl. Acad. Sci. USA 95, 7299-7304 (1998).

12. R. L. Kingston, N. H. Olson, and V. M. Vogt, The organization of mature Rous sarcoma virus as studied by cryoelectron microscopy, J. Struct. Biol. 136, 67-80 (2001).

13. F. Yu, S. M. Joshi, Y. M. Ma, R. L. Kingston, M. N. Simon, and V. M. Vogt, Characterization of Rous sarcoma virus Gag particles assembled in vitro, J. Virol. 75, 2753-2764 (2001).

14. Y. Talmon, B. V. Prasad, J. P. Clerx, G. J. Wang, W. Chiu, and M. J. Hewlett, Electron microscopy of vitrified-hydrated La Crosse virus, J. Virol. 61, 2319-2321 (1987).

15. G. J. Wang, M. Hewlett, and W. Chiu, Structural variation of La Crosse virions under different chemical and physical conditions, Virology 184, 455-459 (1991).

16. Y. Hosaka and T. Watabe, Cryoelectron microscopy of vitrified Sendai virions, J. Virol. Methods. 22, 347349 (1988).

17. B. W. Neuman, B. D. Adair, J. W. Burns, R. A. Milligan, M. J. Buchmeier, and M. Yeager, Complementarity in the supramolecular design of arenaviruses and retroviruses revealed by electron cryomicroscopy and image analysis, J. Virol. 79, 3822-3830 (2005).

18. M. R. Macnaughton, H. A. Davies, and M. V. Nermut, Ribonucleoprotein-like structures from coronavirus particles, J. Gen. Virol. 39, 545-549 (1978).

19. D. Escors, J. Ortego, H. Laude, and L. Enjuanes, The membrane M protein carboxy terminus binds to transmissible gastroenteritis coronavirus core and contributes to core stability, J. Virol. 75, 1312-1324 (2001) 